\title{
IgG antibodies to synthetic GPI are biomarkers of immune-status to both Plasmodium falciparum and Plasmodium vivax malaria in young children
}

Camila T. França ${ }^{1,2^{*}}$, Connie S. N. Li Wai Suen ${ }^{1,2}$, Amandine Carmagnac $^{3}$, Enmoore Lin ${ }^{4}$, Benson Kiniboro ${ }^{4}$, Peter Siba ${ }^{4}$, Louis Schofield ${ }^{1,2,5}$ and Ivo Mueller ${ }^{1,2,6,7}$

\begin{abstract}
Background: Further reduction in malaria prevalence and its eventual elimination would be greatly facilitated by the development of biomarkers of exposure and/or acquired immunity to malaria, as well as the deployment of effective vaccines against Plasmodium falciparum and Plasmodium vivax. A better understanding of the acquisition of immunity in naturally-exposed populations is essential for the identification of antigens useful as biomarkers, as well as to inform rational vaccine development.

Methods: ELISA was used to measure total lgG to a synthetic form of glycosylphosphatidylinositol from P. falciparum (PfGPI) in a cohort of 1-3 years old Papua New Guinea children with well-characterized individual differences in exposure to $P$. falciparum and $P$. vivax blood-stage infections. The relationship between lgG levels to PfGPI and measures of recent and past exposure to $P$. falciparum and $P$. vivax infections was investigated, as well as the association between antibody levels and prospective risk of clinical malaria over 16 months of follow-up.

Results: Total IgG levels to PfGPI were low in the young children tested. Antibody levels were higher in the presence of $P$. falciparum or P. vivax infections, but short-lived. High lgG levels were associated with higher risk of $P$. falciparum malaria (IRR 1.33-1.66, $P=0.008-0.027$ ), suggesting that they are biomarkers of increased exposure to $P$. falciparum infections. Given the cross-reactive nature of antibodies to PfGPI, high IgG levels were also associated with reduced risk of $P$. vivax malaria (IRR 0.65-0.67, $P=0.039-0.044$ ), indicating that these antibodies are also markers of acquired immunity to P. vivax.

Conclusions: This study highlights that in young children, IgG to PfGPI might be a useful marker of immune-status to both P. falciparum and P. vivax infections, and potentially useful to help malaria control programs to identify populations at-risk. Further functional studies are necessary to confirm the potential of PfGPI as a target for vaccine development.
\end{abstract}

Keywords: Plasmodium falciparum, Plasmodium vivax, Malaria elimination, IgG antibody, Biomarker of exposure, GPI, Glycosylphosphatidylinositol, Clinical malaria, Protection, Exposure

\footnotetext{
*Correspondence: franca.c@wehi.edu.au

1 Population Health and Immunity Division, Walter and Eliza Hall Institute,

Parkville, VIC, Australia

Full list of author information is available at the end of the article
} 


\section{Background}

Despite several countries having reduced malaria incidence by more than $75 \%$, and a reduction in mortality by $48 \%$ globally, more than 3 billion people are still at risk of contracting malaria and some 438,000 deaths still occur every year [1]. Current malaria control and elimination efforts would be greatly enhanced by the development of novel and more sensitive surveillance tools. For instance, serological markers that can be used to estimate exposure to malaria parasites and/or indicate a person's immune status would help to identify populations at risk, and to direct resources to areas in more need [2-4]. Additionally, the development and deployment of highly efficacious vaccines against the two major malaria parasites, Plasmodium falciparum and Plasmodium vivax, would certainly accelerate malaria elimination $[2,5]$.

Identifying optimal antigenic targets for evaluating exposure or for vaccine development, however, remains a huge challenge due to the complexity of malaria parasites biology and epidemiology [6]. As the dynamics of antibody acquisition and maintenance vary based on exposure intensity, which serologic markers are informative of exposure or immunity is likely to differ by age group and transmission setting $[4,7,8]$. A better understanding of the human immune responses to malaria parasites is thus essential for biomarker discovery, and very useful in guiding rational vaccine design $[4,7,8]$. To date, relatively little is known about the early acquisition and role of anti-Plasmodium spp. antibodies in young children, how such responses compare to responses in older children/adults, or those from different transmission intensity areas [7-11]. The investigation of antigenic targets and their potential as vaccine candidates or biomarkers of exposure in naturally exposed populations has been mainly restricted to $P$. falciparum and very few $P$. vivax merozoite proteins [7-11].

In malaria parasites, glycosylphosphatidylinositol (GPI) is a glycolipid highly conserved across different species [12]. In Plasmodium spp., GPI can be found both free and as an anchor sustaining many proteins on the parasite's membrane, including merozoite surface and rhoptry proteins, as well as many other vaccine candidates and proteins of unknown function [12]. In humans, GPI is known to induce strong humoral response, promote the expression of genes of pro-inflammatory compounds (including tumour-necrosis factor (TNF), interleukin-1 [IL-1] and IL-12), nitric oxide, and adhesion molecules on the surface of the vascular endothelium, which can be recognized by $P$. falciparum erythrocyte membrane protein 1 (PfEMP1), contributing to the development of anaemia and severe malaria $[13,14]$.
It has been consistently demonstrated that GPIs purified from $P$. falciparum are recognized by plasma/serum from people living in malaria-endemic areas however, the quality of GPIs purified from $P$. falciparum might have led to controversial results $[15,16]$. Cross-reactivity between antibodies raised against $P$. falciparum GPI and $P$. vivax is expected, as despite having a high complexity that allows various chemical modifications and high functional diversity, the core of the GPI glycan structure is evolutionary highly conserved in different species [17]. Only limited structural variability (in fatty-acid composition or glycosylation) or antigenic variation have been described [18-21] in comparison to the many allelic polymorphisms identified in merozoite surface proteins [22-24], and the consequent high antigenic variation [25-27].

To date, the association between the levels of antibodies to GPI and the risk of malaria clinical disease remains poorly explored. To address this gap, this study aimed to measure total IgG levels to a synthetic glycan corresponding to $P$. falciparum GPI (PfGPI) in a cohort of children aged 1-3 years from Papua New Guinea (PNG), exploring the associations between antibody levels and prospective risk of malaria. Individual differences in exposure to Plasmodium spp. blood-stage infections have been well characterized by molecular genotyping [28, 29], and children have been shown to had acquired immunity to $P$. vivax, but no yet to P. falciparum [28-30]. The potential use of IgG to $P f G P I$ as a serological biomarker of immune status to both $P$. falciparum and $P$. vivax parasites was investigated.

\section{Methods}

\section{Antigen}

The synthetic glycan PfGPI described by Schofield et al. [31] was used. As the glycan was conjugated to bovine serum albumin (BSA), BSA alone was included as a control.

\section{Study samples}

Antibody reactivity to PfGPI in naturally exposed individuals was assessed in samples from a longitudinal cohort of 264 children (1-3 years old) undertaken in Ilaita, East Sepik Province, PNG [30]. Children were enrolled between March and September 2006, and followed for up to 16 months. Blood samples were collected every 8 weeks and at episodes of febrile illness. All $P$. falciparum and $P$. vivax infections were genotyped, allowing the determination of the incidence of genetically distinct blood-stage infections acquired during follow-up (i.e. the molecular force of blood-stage infections, molFOB) [28, 29]. Paired samples collected at cohort follow-up start 
and end from 223 children were included in the present study (median age 1.8, IQR 1.3-2.5 years).

\section{Antibody measurement}

Total IgG was measured using an enzyme-linked immunosorbent assay (ELISA). Nunc 96-well plates (Thermo Scientific) were coated with GPI conjugated to BSA or BSA alone diluted to $10 \mathrm{ng} /$ well in phosphate-buffered saline (PBS) $\mathrm{pH} 7.2$, and incubated overnight at $4{ }^{\circ} \mathrm{C}$. The next day, the plates were washed 3 times in PBS and blocked with PBS $+5 \%$ milk for $1 \mathrm{~h}$ at $37{ }^{\circ} \mathrm{C}$. Plates were then washed 3 times in PBS $+0.05 \%$ Tween-20, and plasma samples from PNG children and controls diluted $1: 125$ in PBS $+1 \%$ milk $+0.05 \%$ Tween-20 were assayed in duplicate, with incubation overnight at $4{ }^{\circ} \mathrm{C}$. On the third day, plates were washed 5 times in PBS $+0.05 \%$ Tween-20 and the secondary antibody horseradish peroxidase-conjugated mouse anti-human IgG (Southern biotech) diluted $100 \mathrm{ng} /$ well in PBS $+1 \%$ milk $+0.05 \%$ Tween-20 was added, followed by incubation for $2 \mathrm{~h}$ at room temperature. Finally, plates were washed 5 times in $\mathrm{PBS}+0.05 \%$ tween and TMB peroxidase substrate (KPL) added and incubated for $1 \mathrm{~min}$ and $30 \mathrm{~s}$ until colour developed. $1 \mathrm{M}$ phosphoric acid (Sigma) was used to stop the reaction and absorbance was read at $450 \mathrm{~nm}$. Plasma from seven Australian adults, and a serial dilution of a plasma pool from hyper-immune PNG adults were included as negative and positive controls, respectively. Paired samples from the same individual collected at study start and end were run on the same plate.

\section{Statistical analysis}

Background values due to reactivity to BSA were subtracted and duplicate wells averaged. Associations with parasite density were determined using Spearman's rank correlation. Optical density (OD) values were $\log _{10}$-transformed and differences in mean antibody levels by age, infection status, and between samples collected at start and end of follow-up were assessed using ANOVA or 2-tailed $t$ tests (paired when necessary). Negative binomial generalized estimating equation (GEE) models with exchangeable correlation structure and semi-robust variance estimator were used to analyze the relationship between antibodies to PfGPI and prospective risk of $P$. falciparum and $P$. vivax episodes (defined as axillary temperature $\geq 37.5{ }^{\circ} \mathrm{C}$ or history of fever in the preceding $48 \mathrm{~h}$ with a concurrent parasitaemia $>2500$ and $>500 P$. falciparum and $P . v i v a x / \mu \mathrm{L}$, respectively) over the 16 months of follow-up [11]. In order to investigate this, antibody levels were classified into tertiles (cut-off values are given on Table 1), and analyses carried out comparing the incidence rate ratio (IRR) of clinical malaria in those with medium and high antibody levels versus low. Children were considered at risk from the first day after the blood sample for active follow-up was taken. For each child, the molFOB was calculated as the number of new blood-stage genetically distinct $P$. falciparum or $P$. vivax clones acquired/year-at-risk, and squareroot transformed for a better fit $[28,29]$. Adjustments were made for seasonal trends, village of residency, age, haemoglobin levels, Gerbich blood type, and molFOB.

Table 1 Seroprevalence of IgG antibodies to PfGPI in Papua New Guinean children

\begin{tabular}{lll}
\hline & $\begin{array}{l}\text { Study start } \\
\text { lgG level }{ }^{\mathbf{a}} \text { in children (\% of adult levels) }\end{array}$ & Study end \\
\hline Geometric mean & $0.096(13.87)$ & $0.108(15.62)$ \\
$95 \% \mathrm{Cl}$ & $0.083(11.99)$ & $0.093(13.44)$ \\
& $0.110(15.90)$ & $0.127(18.35)$ \\
Cut-offa low antibody group & $0.061(8.75)$ & \\
Cut-offa medium antibody group & $0.135(19.53)$ & \\
\hline & Study start & Study end \\
& Prevalence in children (\%) & \\
\hline$\%$ of adult levels (cut-offa) & & \\
$1 \%(0.007)$ & $223(100)$ & $223(100)$ \\
$5 \%(0.035)$ & $200(89.67)$ & $196(87.89)$ \\
$10 \%(0.069)$ & $133(59.64)$ & $71(31.84)$ \\
$25 \%(0.173)$ & $55(24.66)$ & $36(16.14)$ \\
$50 \%(0.346)$ & $26(11.66)$ & \\
\hline
\end{tabular}

$P$ values from paired 2-tailed t tests or Chi squared tests. $P<0.05$ were deemed statistically significant

95\% Cl 95\% confidence interval

a Optical density at $450 \mathrm{~nm}$ 
All analyses were performed using STATA version 12 (StataCorp).

\section{Results}

\section{IgG antibodies to PfGPI in young PNG children}

IgG seroprevalence to PfGPI was relatively low at the study start. It was assumed that the pooled serum from immune PNG adults represented the highest antibody levels to PfGPI achievable under natural exposure and, therefore, by comparison with IgG levels observed in PNG children, the number of children that had already achieved IgG levels that were $>50,>25$ or $>10 \%$ of the maximum adult levels (Table 1 ) was determined. At this time point, only 11.7 and $59.6 \%$ of the PNG children had acquired IgG levels that were $>50$ and $>10 \%$ of the immune adult levels (Table 1).

Overall, although the 1-3 years old children tested in this study had acquired low levels of antibodies to PfGPI, the response observed was directed and significantly higher to PfGPI (mean OD to GPI after BSA subtraction $=0.18,95 \% \mathrm{CI} 0.14-0.21)$ than to the BSA tag alone (mean OD to BSA alone $=0.08,95 \%$ CI 0.07 $0.09, \mathrm{P}=0.009)$ (Additional file 1 ).

\section{Influence of age and exposure to malaria parasites}

At study start, there was no association between age and IgG levels to PfGPI $(\mathrm{P}=0.53)$ (Table 2). An increase in IgG levels with age was only observed in children $>24$ months old and free of $P$. falciparum infection (detected by PCR) at the moment of sample collection $(\mathrm{P}=0.009)$, suggesting that antibodies to PfGPI are reflective of recent malaria infections. Children with a current $P$. falciparum $(\mathrm{P}=0.084, \mathrm{n}=112)$ (although only moderately), $P$. vivax $(\mathrm{P}=0.036, \mathrm{n}=125)$ or mixed infection $(P$. falciparum $+P$. vivax $)(\mathrm{P}=0.004, \mathrm{n}=65)$ had higher antibody levels than children infection-free (Table 2). There were however, no associations between IgG levels and $P$. falciparum or $P$. vivax parasite densities $($ rho $=0.09, \mathrm{P}>0.18)$. Children with the highest IgG levels were also more likely to have a P. falciparum (Odds

Table 2 Influence of age and exposure on antibody levels to PfGPI in Papua New Guinean children

\begin{tabular}{|c|c|c|c|c|c|c|}
\hline & \multicolumn{3}{|c|}{ P. falciparum } & \multicolumn{3}{|c|}{ P. vivax } \\
\hline & $\mathbf{n}$ & Geom mean $(95 \% \mathrm{Cl}) *$ & $P$ value & $\mathbf{n}$ & Geom mean $(95 \% \mathrm{Cl})^{*}$ & $P$ value \\
\hline \multicolumn{7}{|l|}{ Age (months) } \\
\hline \multicolumn{7}{|l|}{ All children } \\
\hline $12-17$ & 81 & $0.091(0.074-0.111)$ & 0.53 & & & \\
\hline $18-23$ & 49 & $0.083(0.061-0.114)$ & & & & \\
\hline $24-29$ & 38 & $0.117(0.080-0.170)$ & & & & \\
\hline $30-35$ & 45 & $0.108(0.075-0.155)$ & & & & \\
\hline $36-41$ & 10 & $0.081(0.032-0.204)$ & & & & \\
\hline \multicolumn{7}{|l|}{ PCR- } \\
\hline $12-17$ & 55 & $0.076(0.060-0.097)$ & 0.009 & 37 & $0.067(0.051-0.087)$ & 0.221 \\
\hline $18-23$ & 22 & $0.053(0.036-0.078)$ & & 19 & $0.074(0.043-0.128)$ & \\
\hline $24-29$ & 14 & $0.160(0.079-0.323)$ & & 18 & $0.126(0.086-0.185)$ & \\
\hline $30-35$ & 16 & $0.113(0.062-0.207)$ & & 19 & $0.083(0.047-0.146)$ & \\
\hline $36-41$ & 4 & $0.155(0.014-1.737)$ & & 5 & $0.087(0.049-0.156)$ & \\
\hline \multicolumn{7}{|l|}{ PCR+ } \\
\hline $12-17$ & 26 & $0.131(0.090-0.192)$ & 0.43 & 44 & $0.118(0.088-0.157)$ & 0.69 \\
\hline $18-23$ & 27 & $0.120(0.078-0.186)$ & & 30 & $0.090(0.060-0.133)$ & \\
\hline $24-29$ & 24 & $0.097(0.062-0.153)$ & & 20 & $0.109(0.056-0.210)$ & \\
\hline $30-35$ & 29 & $0.106(0.065-0.170)$ & & 26 & $0.131(0.080-0.215)$ & \\
\hline $36-41$ & 6 & $0.053(0.018-0.154)$ & & 5 & $0.075(0.007-0.773)$ & \\
\hline \multicolumn{7}{|l|}{ Infection status } \\
\hline PCR- & 111 & $0.085(0.070-0.103)$ & 0.084 & 98 & $0.081(0.067-0.098)$ & 0.036 \\
\hline PCR+ & 112 & $0.108(0.088-0.133)$ & & 125 & 0.109 (0.089-0.134) & \\
\hline Infection free & 51 & $0.061(0.047-0.077)$ & 0.004 & & & \\
\hline Pf and Pv co-infected & 65 & $0.107(0.079-0.144)$ & & & & \\
\hline
\end{tabular}

Geom mean, geometric mean; $n$, number; $95 \%$ Cl, 95\% confidence interval; Pf, Plasmodium falciparum; Pv, Plasmodium vivax

* Optical density at $450 \mathrm{~nm}$. IgG levels were log 10 transformed and $P$ values calculated using two sample $t$ tests or ANOVA. P $<0.05$ were considered significant 
ratio $[\mathrm{OR}] 2.77,95 \% \mathrm{CI} 1.43-5.38, \mathrm{P}=0.003)$ or $P$. vivax (OR 1.91, 95\% CI 0.99-3.70, $\mathrm{P}=0.055$ ) infection in the following 2 months.

Due to the small age range in this cohort, however, the number of genetically distinct blood-stage parasites that each child acquired over time (i.e. the molFOB) is a better proxy for exposure to malaria than age alone $[28,29]$. Thus, calculating life-time exposure as a product of age and molFOB, an increase in IgG levels to PfGPI was found with increasing life-time exposure to $P$. vivax blood-stage infections (Spearman's rho $=0.15$, $\mathrm{P}=0.026$ ), with stronger effects observed in children free of $P$. vivax infections at sample collection (rho $=0.23$, $\mathrm{P}=0.026)$.

The risk of malaria infection was heterogeneously distributed across the different villages where the study was conducted [30]. Anti-PfGPI antibody levels did reflect such differences, and IgG levels were significantly different when grouping individuals by village of residence $(\mathrm{P}=0.025)$ (Additional file 2). Individuals living in the villages Ilaita 2 and $6(\mathrm{P}=0.06-0.007, \mathrm{n}=10$ and 12 , respectively), and Sunuhu $1(\mathrm{P}=0.004, \mathrm{n}=36)$ had higher IgG levels to PfGPI. These regional differences were significant if children were co-infected $(\mathrm{P}=0.048)$ or infected with $P$. vivax $(\mathrm{P}=0.001)$, but not in the absence of infection $(P>0.3)$ (Additional file 2). Similar differences in antibody levels to P. falciparum AMA1 and MSP2 within these regions have been described [7].

\section{Anti-PfGPI antibodies and morbidity}

Anti-PfGPI antibodies were strongly associated with morbidity. IgG levels were negatively correlated with haemoglobin levels (rho $=-0.18, \mathrm{P}=0.007$ ), (Fig. 1a), and elevated IgG levels were present in those with a palpable, enlarged spleen $(\mathrm{P}=0.037)$ (Fig. 1b). Similarly, children with a current clinical episode by $P$. falciparum $(\mathrm{P}=0.010)$, but not $P . \operatorname{vivax}(\mathrm{P}=0.21)$, had higher IgG levels than uninfected or asymptomatically infected children (Fig. 1c). Interestingly, there was a strong association between anti-PfGPI antibodies and the children's Gerbich blood type. The few Gerbich homozygote children had the highest IgG levels $(P=0.001)$ (Fig. 1d).

\section{IgG antibodies to PfGPI and prospective risk of falciparum-malaria}

Over the 16 months of follow-up, each child in the subgroup tested had an average of 1.54 (95\% CI 1.38-1.73) P. falciparum clinical episodes with $>2500$ parasites/ $\mu \mathrm{L} /$ year-at-risk. Following adjustment for age, seasonal and spatial differences in malaria transmission, Gerbich blood type and haemoglobin levels, high levels of IgG to PfGPI were associated with an increased risk of having $P$. falciparum-malaria (Incidence rate ratio for high versus low group $\left[\operatorname{IRR}_{\mathrm{H}}\right]$ 1.36, $\mathrm{P}=0.027$ ) (Fig. 2; Additional file 3). The risk increased for clinical episodes with higher parasite densities: $>10,000$ parasites/ $\mu \mathrm{L}\left(\mathrm{IRR}_{\mathrm{M}}\right.$ $1.35, \mathrm{P}=0.044$ and $\left.\mathrm{IRR}_{\mathrm{H}} 1.42, \mathrm{P}=0.028\right)$, $>50,000$ parasites $/ \mu \mathrm{L}\left(\mathrm{IRR}_{\mathrm{M}} 1.80, \mathrm{P}=0.004\right)$ (Fig. 2; Additional file 3). Once adjusted for individual differences in exposure to $P$. falciparum infections (molFOB), the strength and significance of the associations were decreased or became no longer significant $\left(>2500\right.$ : $\mathrm{IRR}_{\mathrm{H}} 1.26, \mathrm{P}=0.07 ;>10,000$ : $\mathrm{IRR}_{\mathrm{M}} 1.17$, and $\mathrm{IRR}_{\mathrm{H}} 1.29, \mathrm{P}=0.08-0.26 ;>50,000: \mathrm{IRR}_{\mathrm{M}}$ $1.59, \mathrm{P}=0.018$ ) (Additional file 3), indicating that in this age group, high IgG levels to PfGPI are markers of children who had higher exposure to $P$. falciparum parasites, which consequently led to a higher risk of having clinical disease.

\section{IgG antibodies to PfGPI and prospective risk of vivax-malaria}

The average of clinical episodes caused by $P$. vivax (> 500 parasites $/ \mu \mathrm{L}$ ) was 1.22 (95\% CI $1.05-1.42)$ per year-at-risk. In contrast to that observed for $P$. falciparum-malaria, after adjustments for confounders and differences in individual exposure to $P$. vivax blood-stage infections, high IgG levels to PfGPI were associated with a modestly reduced risk of vivax-malaria $\left(\operatorname{IRR}_{\mathrm{H}} 0.72\right.$, $\mathrm{P}=0.049$ ) (Fig. 2; Additional file 3). As for P. falciparum, the associations with protection tended to be stronger for clinical episodes with higher parasite density, although not of statistical significance given the reduced power ( $>2000$ parasites $/ \mu \mathrm{LIRR}_{\mathrm{H}} 0.68, \mathrm{P}=0.057$; $>10,000$ parasites $/ \mu \mathrm{L} \operatorname{IRR}_{\mathrm{H}} 0.59, \mathrm{P}=0.094$ ) (Fig. 2; Additional file 3). These results indicate that high levels of IgG to PfGPI in this age group are also markers of acquired immunity to P. vivax.

\section{Antibodies to PfGPI after 16 months}

At the end of the 16 months of follow-up, 64.6 and $16.1 \%$ of the children had reached IgG levels that were $>10$ and $>50 \%$ of the IgG levels observed in the adult immune pool (Table 1). Although slightly higher, antibody levels were very similar $($ rho $=0.57, \mathrm{P}<0.001)$ and this seroprevalence was not statistically different than the observed at the study start $(\mathrm{P}>0.17)$, suggesting that in this age group, anti-PfGPI antibodies are short-lived or unstable.

At the end of the study, there was no association between IgG levels and age ( $P>0.18)$, life-time exposure $(\mathrm{P}>0.20), P$. falciparum or $P$. vivax infection status $(P>0.05)$ (Additional file 4). No difference in IgG levels was observed between children who experienced a clinical episode in the last 2 months and those who did not $(\mathrm{P}>0.18)$, or by the number of clinical episodes that each child had over the follow-up period for P. falciparum or 

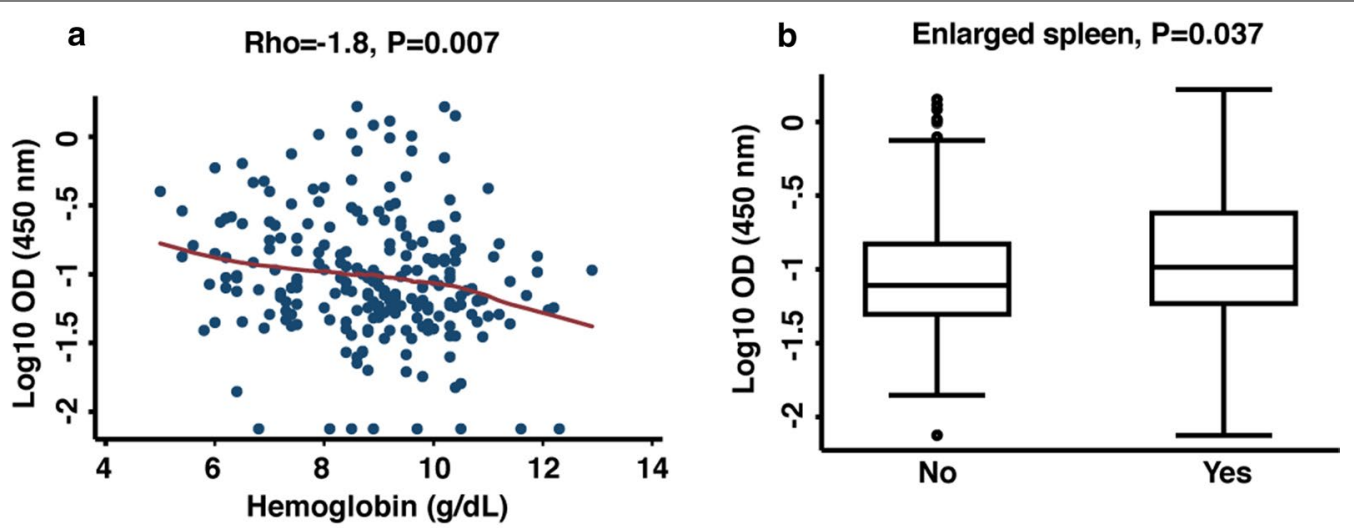

C
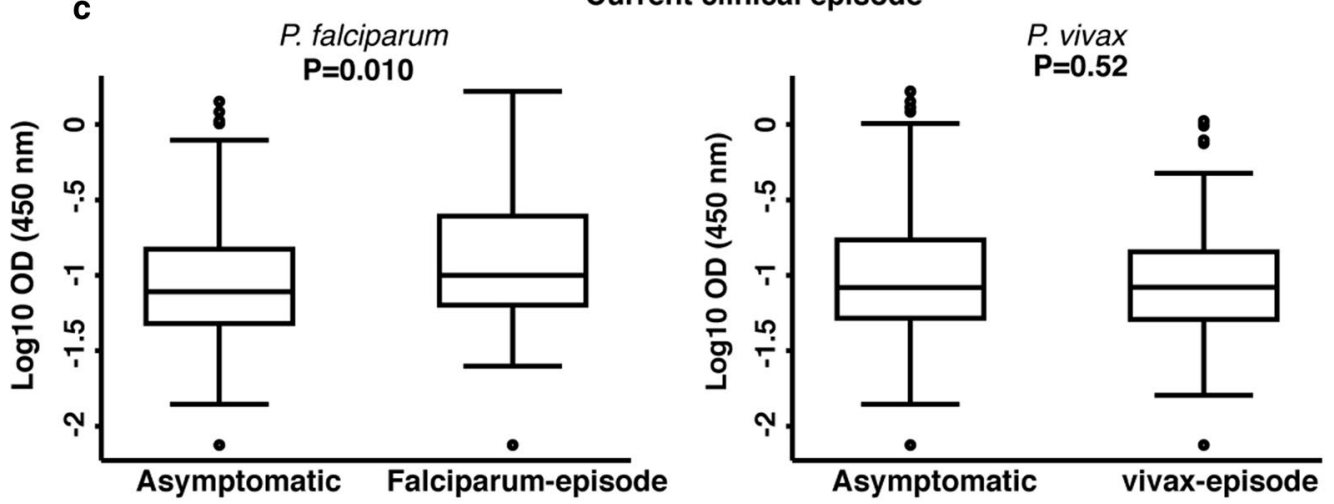

d Gerbich blood type, $P=0.001$

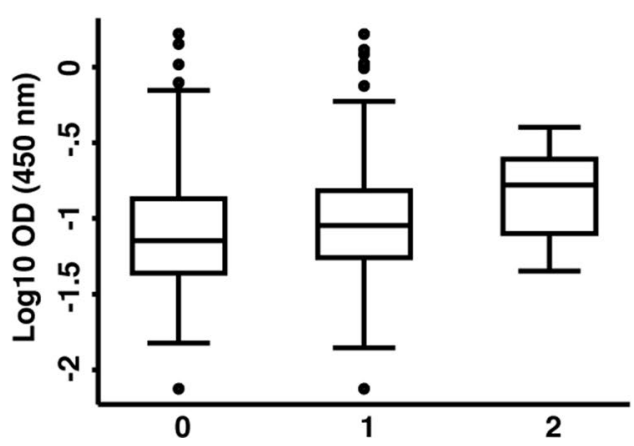

Fig. $1 \mathrm{lgG}$ to PfGPI and clinical symptoms in Papua New Guinean children. a Scatterplot of total lgG levels (optical density at $450 \mathrm{~nm}$ ) versus haemoglobin levels $(\mathrm{g} / \mathrm{dL})(\mathrm{n}=223)$ with a Lowess smoothed fitted curve. P values and rho are from Spearman's rank correlation. Box plots show median IgG levels (black bar), minimum and maximum (whiskers) and outliers (open circles) by b presence of enlarged spleen ( $n=59) ; \mathbf{c} c u r-$ rent clinical episode of any density by P. falciparum $(n=65)$ or P. vivax $(n=70)$; d Gerbich blood type $1=$ wild-type $(n=83), 2=$ heterozygote $(n=111), 3=$ homozygote $(n=29)$. $P$ values are from ANOVA or 2 sample $t$ tests

P. $\operatorname{vivax}(\mathrm{P}>0.31)$ (Additional file 4). Similarly, there was no difference in IgG levels at the end of the follow-up between children who did and who did not experience severe malaria during follow-up $(\mathrm{n}=24, \mathrm{P}=0.97)$.

At the end of the study, the only factor associated with differences in antibody levels was village of residency for those currently co-infected $(\mathrm{P}=0.046)$ or with a $P$. vivax infection $(P=0.008)$ (Additional file 2).

\section{Discussion}

A better understanding of the acquisition of immunity to malaria parasites in different age groups and transmission settings is essential for the identification of antigens useful as biomarkers of exposure/immunity, or with potential for vaccine development-especially for $P$. vivax, since a continuous in vitro culture system is still inexistent $[4,5]$. In the present study, antibody levels to a 


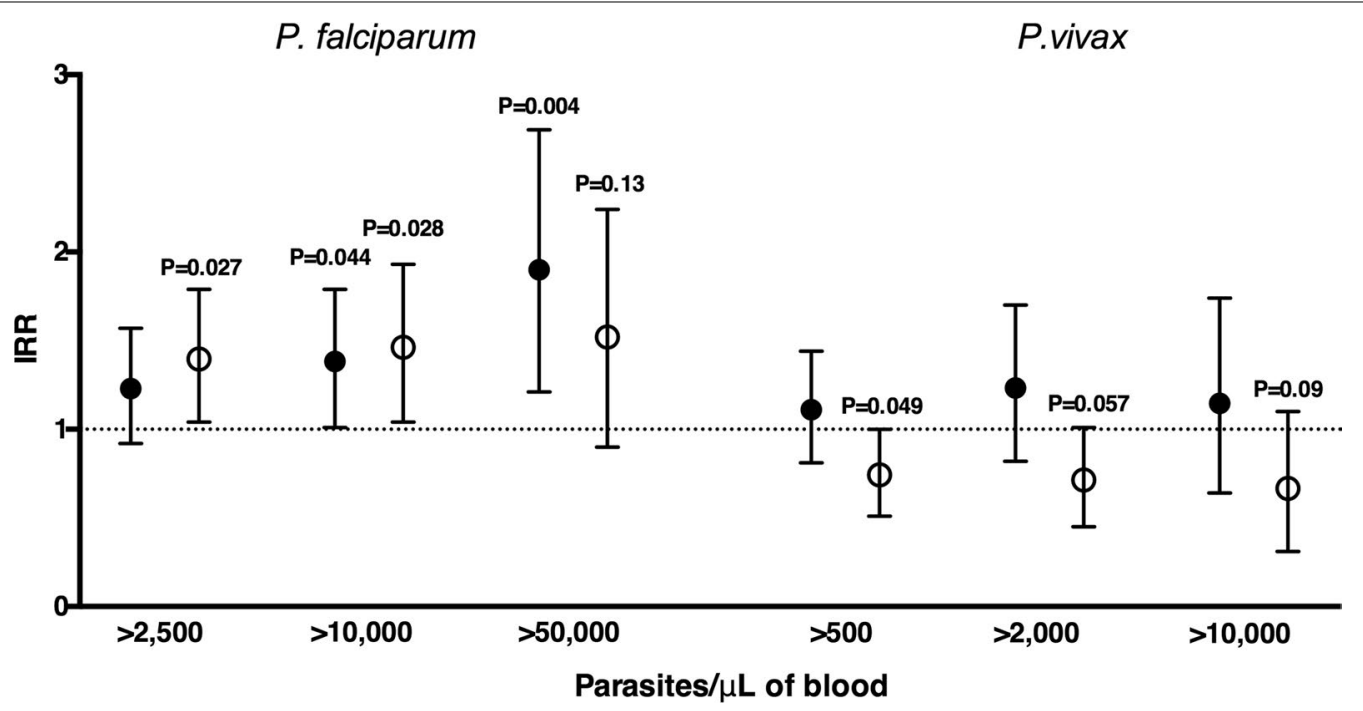

Fig. 2 lgG to PfGPI and risk of falciparum and vivax-malaria in Papua New Guinean children. Data are plotted as incidence rate ratios and $95 \%$ confidence intervals over 16 months of follow-up, adjusted for age, season, village of residency, haemoglobin levels and Gerbich blood type ( $n=223$ ). Black and white circles represent children with medium and high antibody levels, respectively. Clinical malaria was defined as axillary temperature $\geq 37.5^{\circ} \mathrm{C}$ or history of fever in the preceding $48 \mathrm{~h}$ with a current P. falciparum parasitemia of $>2500(n=383) ;>10,000(n=315) ;$ and $>50,000$

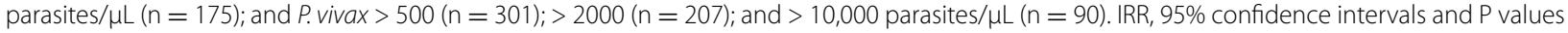
are from negative binomial GEE models

synthetic glycan correspondent to PfGPI [31] was measured in a cohort of children 1-3 years old from PNG [30], exploring the associations between antibody levels and risk of $P$. falciparum and $P$. vivax-malaria.

Despite the very high transmission intensity in East Sepik Province when the cohort study was conducted [30], seroprevalence of antibodies to PfGPI was low in this age group. Similar low seroprevalence have been described in children $<6$ years from Madang Province in PNG [32], as well as in Indonesia [33], Kenya [18] and Gambia [34]. One explanation for this is the low ability of the immune system of very young children $(<2$ years old) in producing antibodies against carbohydrate antigens [35]. This also suggests that the majority of GPI that the immune system has access to and thus can produce antibodies to is the free form, rather than the form that anchors proteins to the parasite membrane. If physically attached to their GPI anchors, parasite surface proteins might be expected to provide $\mathrm{T}$ cell help for anti-GPI antibody production [15]. Although not observed in the young children included in the study, seroprevalence and magnitude of antibody responses to PfGPI have been described to increase with age and decline with parasite density in PNG [32] and Kenyan adults [18].

For the young children included in this study, recent $P$. falciparum and $P$. vivax infections were the main determinant of antibody levels to GPI. The rapid although transient peaks in antibody levels in the presence of a current infection might suggest that they are generated by the differentiation of naive B-cells into short-lived plasma cells driven by the concurrent infection rather than by long-lived plasma cells generated from previous infections, as previously described for malarial protein antigens [36]. Given the absence of peptide epitopes for conventional $\mathrm{T}$ cells, antibodies to free GPI are likely to be $\mathrm{T}$ cell-independent during the first malaria infections [15]. Although they can stimulate antigen-specific $B$ cells, memory is not generated, and accessory cells (e.g. macrophages and dendritic cells) and co-stimulatory signals (e.g. IL-1) are thus required for an effective immune response [37]. Later with increasing exposure, or if attached to an immunogenic carrier, however, GPI might be taken up by follicular B cells, be processed and presented on cell surface major histocompatibility complex class II (MHCII) molecules, where they may engage peptide-specific T cells $[15,35]$. Memory B cells can thus be generated during this $\mathrm{T}$ cell-dependent process, and be re-activated upon future stimulation [35].

Children with homozygote Gerbich blood type (Gerbich negative) had higher antibody levels to PfGPI than heterozygote or wild type children. The Gerbich antigen is expressed on glycophorins C (GPC) and D (GPD) [38], and both GPC/D interact with the $4.1 \mathrm{R}$ protein complex and contribute to the stability of the erythrocyte membrane [38, 39]. A high incidence of Gerbich negative in PNG been hypothesized as an advantage against 
infection and severe malaria $[40,41]$. While it was found that deletion of the exon 3 result in Gerbich negativity and make $P$. falciparum unable to invade erythrocytes using the erythrocyte binding protein 140 [EBA140] [39, 42], to date, clinical studies have not been able to show a consistent association between risk of malaria and this phenotype [43-45]. Further in-depth studies will be required to elucidate whether the interaction between Gerbich genotype, reduced parasite invasion and slower parasite growth result in increased host immuneresponses (including to $P f G P I$ ), and whether this may indeed combine to provide protection against $P$. falciparum or P. vivax malaria [46].

In young PNG children, high antibody levels to PfGPI were associated with higher risk of $P$. falciparum malaria. In contrast, they were also associated with reduced risk of $P$. vivax malaria. This accurately reflects the different levels of naturally acquired immunity to the two species in this cohort: while in these children incidence of $P$. vivax episodes significantly decreases starting in the 2nd year of life, the burden of $P$. falciparum infection continues to increase until the 4th year of life [30]. This difference is related to a significantly higher exposure to $P$. vivax than $P$. falciparum blood-stage infections, i.e. $P$. vivax molFOB was considerably higher than $P$. falciparum molFOB (14 versus 5.5 parasite clones/child/yearat-risk, respectively). This high number of $P$. vivax clones that infect children in early childhood thus contribute to a very rapid acquisition of immunity to clinical $P$. vivax malaria, not yet reached for $P$. falciparum $[29,30]$. Acquisition of immunity to $P$. falciparum in high transmission settings such as PNG is achieved a number of years later ( 10 years old) with increasing exposure to P. falciparum infections $[7,8]$. Anti-PfGPI antibodies in this age group seem to be an accurate reflection of the children's current immune-status to both $P$. falciparum and $P$. vivax malaria, acting as both a biomarker of increased risk of $P$. falciparum, able to identify individuals with the highest level of exposure to $P$. falciparum recent infections, as well as a biomarker of acquired immunity to $P$. vivax.

In 2002, a study in rodent models firstly showed that antibodies raised against PfGPI were able to delay mortality by Plasmodium berghei, demonstrating proof of concept for a GPI-based anti-toxic malaria vaccine [31]. The antagonists of GPI-mediated signaling and murine monoclonal antibodies against PfGPIs were shown to be able to block the induction of toxic responses, also suggesting that GPI-based therapy is possible $[47,48]$. In more recent studies, GPI was found to be present across all stages of the malaria parasites life cycles. Furthermore, in a pre-clinical evaluation of a GPI-based vaccine in $P$. berghei models, the vaccine showed efficacy in sporozoite challenges, was able to reduce parasite replication and transmission to mosquitoes (unpublished data, Schofield.) Altogether, these findings suggest that a GPI vaccine may be able to prevent both blood-stage and liver infections, disease and block transmission of parasite from human to mosquito, thus acting as a unique carbohydrate multi-stage, multiparasite vaccine. Consistent with this, high levels of the anti-GPI antibodies have been correlated with resistance to clinical symptoms, such as anaemia and fever [18], and lower levels observed among Senegalese adults with cerebral malaria compared to individuals with uncomplicated malaria [49]. Although anti-PfGPI antibodies are shortlived or intermittent in very young children, older children and adults seem to be able to sustain high antibody levels for longer [18, 32-34, 50]. Furthermore, GPI low immunogenicity in young children and can be overcome if the antigen is conjugated to a protein carrier, which can also help stimulation of B-cell memory formation [35]. Future functional studies are now necessary to confirm whether antiPfGPI antibodies contribute to the protection observed against $P$. vivax, or only act as a mirror of the protection conferred by antibodies to other antigenic targets.

This study highlights anti-PfGPI antibodies as a possible biomarker of anti-malaria immunity in very young children. Further studies including older age groups will confirm its utility as a biomarker of immunity for $P$. vivax, and whether they will indeed also reflect acquired immunity to $P$. falciparum.

\section{Conclusions}

The findings of this study highlight IgG to PfGPI as potentially useful serological biomarkers of immune-status in young children to help malaria control programs identify populations at risk. Additional studies including older age groups will confirm the utility of these responses as a biomarker of immunity to $P$. vivax, and whether they will indeed also reflect acquired immunity to $P$. falciparum. Future functional studies are also necessary to confirm whether anti-PfGPI antibodies contribute to the protection observed against $P$. vivax, or only act as a mirror of the protection conferred by antibodies to other antigenic targets.

\section{Additional files}

Additional file 1. Antibody responses to PfGPI and the BSA tag in 1-3 years old children. Box plots show median lgG levels (central bar), minimum and maximum (whiskers) and outliers (grey circles). $n=223$. $P$ value is from t-test.

Additional file 2. IgG to PfGPI by village of residency.

Additional file 3. Association between IgG to PfGPI and protection against clinical malaria in Papua New Guinean children.

Additional file 4. Influence of age and exposure on antibody levels to PfGPI in Papua New Guinean children. 


\section{Authors' contributions}

The following authors contributed with: samples and reagents used in this study - LS, EL, BK, PS; study design — IM, LS; data generation - CTF and AC; statistical analysis-CTF, CSNLWS, IM; manuscript writing-CTF, and IM. All authors read and approved the final manuscript.

\section{Author details}

1 Population Health and Immunity Division, Walter and Eliza Hall Institute, Parkville, VIC, Australia. ${ }^{2}$ Department of Medical Biology, University of Melbourne, Melbourne, VIC, Australia. ${ }^{3}$ Infection and Immunity Division, Walter and Eliza Hall Institute, Parkville, VIC, Australia. ${ }^{4}$ Malaria Immuno-Epidemiology Unit, PNG Institute of Medical Research, Madang, Madang Province, Papua New Guinea. ${ }^{5}$ Australian Institute of Tropical Health and Medicine, James Cook University, Townsville, QLD, Australia. ${ }^{6}$ Malaria Parasites \& Hosts Unit, Department of Parasites \& Insect Vectors, Institut Pasteur, Paris, France. ${ }^{7}$ BarceIona Institute of Global Health (ISGLOBAL), Barcelona, Spain.

\section{Acknowledgements}

We thank all patients and their families for participating in this study, and the large Papua New Guinean field team that assisted in the conduct of the fieldwork. We are grateful to Dr. Danika Hill for providing samples from malarianaive Australian donors and the immune pool from PNG donors used as controls in our ELISA experiments, and Drs. Hayley Joseph and Rhea Longley for helpful comments during manuscript preparation.

\section{Competing interests}

The authors declare that they have no competing interests.

\section{Availability of data and materials}

Data cannot be made publicly available because it would compromise participant privacy and violates the ethical agreement in the informed consent forms. Data is available upon reasonable request by contacting the PNG Medical Research Advisory Committee and the PNG Institute of Medical Research IRB. The contact is Dr.William Pomat, secretary PNG IMR IRB:William.Pomat@pngimr.org.pg.

\section{Ethical statement}

Ethical clearance was obtained from the PNG Medical Research and Advisory Committee (MRAC 05.19), and the Walter and Eliza Hall Institute (HREC 07/07). Written informed consent was obtained from the parents or guardians of all children participating in the cohort study.

\section{Funding}

This study was funded in part by the Southwest Pacific International Centre of Excellence in Malaria Research (NIH Grant U19A1089686"Research to control and eliminate malaria in the Southwest Pacific"), the National Institutes of Health (Al063135), the National Health \& Medical Research Council (\#1021544), the Malaria Elimination Science Alliance (MESA). This work was made possible through Victorian State Government Operational Infrastructure Support and Australian Government NHMRC IRIISS. I.M. is supported by an NHMRC Senior Research Fellowship (\#1043345), C.T.F. is supported by the University of Melbourne-Melbourne International Postgraduate Scholarship (MIPS).

The funders had no role in study design, data collection and analysis, decision to publish, or preparation of the manuscript.

\section{Publisher's Note}

Springer Nature remains neutral with regard to jurisdictional claims in published maps and institutional affiliations.

Received: 2 August 2017 Accepted: 21 September 2017 Published online: 25 September 2017

\section{References}

1. WHO. World malaria report 2015. Geneva: World Health Organization; 2015.

2. Tanner M, Greenwood B, Whitty CJ, Ansah EK, Price RN, Dondorp AM, et al. Malaria eradication and elimination: views on how to translate a vision into reality. BMC Med. 2015;13:167.
3. Cotter C, Sturrock HJ, Hsiang MS, Liu J, Phillips AA, Hwang J, et al. The changing epidemiology of malaria elimination: new strategies for new challenges. Lancet. 2013;382:900-11.

4. King CL, Davies DH, Felgner P, Baum E, Jain A, Randall A, et al. Biosignatures of exposure/transmission and immunity. Am J Trop Med Hyg. 2015;93:16-27.

5. Mueller I, Shakri AR, Chitnis CE. Development of vaccines for Plasmodium vivax malaria. Vaccine. 2015;33:7489-95.

6. Mueller I, Galinski MR, Baird JK, Carlton JM, Kochar DK, Alonso PL, et al. Key gaps in the knowledge of Plasmodium vivax, a neglected human malaria parasite. Lancet Infect Dis. 2009;9:555-66.

7. Stanisic DI, Fowkes FJ, Koinari M, Javati S, Lin E, Kiniboro B, et al. Acquisition of antibodies against Plasmodium falciparum merozoites and malaria immunity in young children and the influence of age, force of infection, and magnitude of response. Infect Immun. 2015;83:646-60.

8. Richards JS, Arumugam TU, Reiling L, Healer J, Hodder AN, Fowkes FJ, et al. Identification and prioritization of merozoite antigens as targets of protective human immunity to Plasmodium falciparum malaria for vaccine and biomarker development. J Immunol. 2013;191:795-809.

9. Fowkes FJ, Richards JS, Simpson JA, Beeson JG. The relationship between anti-merozoite antibodies and incidence of Plasmodium falciparum malaria: a systematic review and meta-analysis. PLoS Med. 2010; 7:e1000218.

10. Cutts JC, Powell R, Agius PA, Beeson JG, Simpson JA, Fowkes FJ. Immunological markers of Plasmodium vivax exposure and immunity: a systematic review and meta-analysis. BMC Med. 2014;12:150.

11. Franca CT, Hostetler JB, Sharma S, White MT, Lin E, Kiniboro B, et al. An antibody screen of a Plasmodium vivax antigen library identifies novel merozoite proteins associated with clinical protection. PLoS Negl Trop Dis. 2016;10:e0004639.

12. Gilson PR, Nebl T, Vukcevic D, Moritz RL, Sargeant T, Speed TP, et al. Identification and stoichiometry of glycosylphosphatidylinositol-anchored membrane proteins of the human malaria parasite Plasmodium falciparum. Mol Cell Proteom. 2006;5:1286-99.

13. Brattig NW, Kowalsky K, Liu X, Burchard GD, Kamena F, Seeberger PH. Plasmodium falciparum glycosylphosphatidylinositol toxin interacts with the membrane of non-parasitized red blood cells: a putative mechanism contributing to malaria anemia. Microbes Infect. 2008;10:885-91.

14. Schofield L, Grau GE. Immunological processes in malaria pathogenesis. Nat Rev Immunol. 2005;5:722-35.

15. Boutlis CS, Riley EM, Anstey NM, de Souza JB. Glycosylphosphatidylinositols in malaria pathogenesis and immunity: potential for therapeutic inhibition and vaccination. Curr Top Microbiol Immunol. 2005:297:145-85.

16. Mbengue B, Niang B, Niang MS, Varela ML, Fall B, Fall MM, et al. Inflammatory cytokine and humoral responses to Plasmodium falciparum glycosylphosphatidylinositols correlates with malaria immunity and pathogenesis. Immun Inflamm Dis. 2016;4:24-34.

17. Swarts BM, Guo Z. Chemical synthesis of glycosylphosphatidylinositol anchors. Adv Carbohydr Chem Biochem. 2012;67:137-219.

18. Naik RS, Branch OH, Woods AS, Vijaykumar M, Perkins DJ, Nahlen BL, et al. Glycosylphosphatidylinositol anchors of Plasmodium falciparum: molecular characterization and naturally elicited antibody response that may provide immunity to malaria pathogenesis. J Exp Med. 2000;192:1563-76.

19. Kamena F, Tamborrini M, Liu X, Kwon YU, Thompson F, Pluschke G, et al. Synthetic GPI array to study antitoxic malaria response. Nat Chem Biol. 2008:4:238-40.

20. Gerold P, Dieckmann-Schuppert A, Schwarz RT. Glycosylphosphatidylinositols synthesized by asexual erythrocytic stages of the malarial parasite, Plasmodium falciparum. Candidates for plasmodial glycosylphosphatidylinositol membrane anchor precursors and pathogenicity factors. J Biol Chem. 1994;269:2597-606.

21. Tamborrini M, Liu X, Mugasa JP, Kwon YU, Kamena F, Seeberger PH, et al. Synthetic glycosylphosphatidylinositol microarray reveals differential antibody levels and fine specificities in children with mild and severe malaria. Bioorgan Med Chem. 2010;18:3747-52.

22. Arnott A, Mueller I, Ramsland PA, Siba PM, Reeder JC, Barry AE. Global population structure of the genes encoding the malaria vaccine candidate, Plasmodium vivax apical membrane antigen 1 (PVAMA1). PLoS Negl Trop Dis. 2013;7:e2506. 
23. Barry AE, Schultz L, Buckee CO, Reeder JC. Contrasting population structures of the genes encoding ten leading vaccine-candidate antigens of the human malaria parasite, Plasmodium falciparum. PLoS ONE. 2009:4:e8497.

24. Tessema SK, Monk SL, Schultz MB, Tavul L, Reeder JC, Siba PM, et al. Phylogeography of var gene repertoires reveals fine-scale geospatial clustering of Plasmodium falciparum populations in a highly endemic area. Mol Ecol. 2015;24:484-97.

25. Barry AE, Trieu A, Fowkes FJ, Pablo J, Kalantari-Dehaghi M, Jasinskas A, et al. The stability and complexity of antibody responses to the major surface antigen of Plasmodium falciparum are associated with age in a malaria endemic area. Mol Cell Proteom. 2011;10(M111):008326.

26. Gray JC, Corran PH, Mangia E, Gaunt MW, Li Q, Tetteh KK, et al. Profiling the antibody immune response against blood stage malaria vaccine candidates. Clin Chem. 2007;53:1244-53.

27. Cole-Tobian JL, Michon P, Biasor M, Richards JS, Beeson JG, Mueller I, et al. Strain-specific duffy binding protein antibodies correlate with protection against infection with homologous compared to heterologous Plasmodium vivax strains in Papua New Guinean children. Infect Immun. 2009;77:4009-17.

28. Mueller I, Schoepflin S, Smith TA, Benton KL, Bretscher MT, Lin E, et al. Force of infection is key to understanding the epidemiology of Plasmodium falciparum malaria in Papua New Guinean children. Proc Natl Acad Sci USA. 2012;109:10030-5.

29. Koepfli C, Colborn KL, Kiniboro B, Lin E, Speed TP, Siba PM, et al. A high force of Plasmodium vivax blood-stage infection drives the rapid acquisition of immunity in Papua New Guinean children. PLoS Negl Trop Dis. 2013;7:e2403.

30. Lin E, Kiniboro B, Gray L, Dobbie S, Robinson L, Laumaea A, et al. Differential patterns of infection and disease with P. falciparum and $P$. vivax in young Papua New Guinean children. PLoS ONE. 2010;5:e9047.

31. Schofield L, Hewitt MC, Evans K, Siomos MA, Seeberger PH. Synthetic $\mathrm{GPI}$ as a candidate anti-toxic vaccine in a model of malaria. Nature. 2002;418:785-9.

32. Boutlis CS, Gowda DC, Naik RS, Maguire GP, Mgone CS, Bockarie MJ, et al. Antibodies to Plasmodium falciparum glycosylphosphatidylinositols: inverse association with tolerance of parasitemia in Papua New Guinean children and adults. Infect Immun. 2002;70:505-57.

33. Hudson Keenihan SN, Ratiwayanto S, Soebianto S, Krisin Marwoto H, Krishnegowda G, et al. Age-dependent impairment of lgG responses to glycosylphosphatidylinositol with equal exposure to Plasmodium falciparum among Javanese migrants to Papua, Indonesia. Am J Trop Med Hyg. 2003:69:36-41.

34. de Souza JB, Todd J, Krishegowda G, Gowda DC, Kwiatkowski D, Riley EM. Prevalence and boosting of antibodies to Plasmodium falciparum glycosylphosphatidylinositols and evaluation of their association with protection from mild and severe clinical malaria. Infect Immun. 2002;70:5045-51.

35. Lucas AH, Apicella MA, Taylor CE. Carbohydrate moieties as vaccine candidates. Clin Infect Dis. 2005;41:705-12.

36. Weiss GE, Traore B, Kayentao K, Ongoiba A, Doumbo S, Doumtabe D, et al. The Plasmodium falciparum-specific human memory B cell compartment expands gradually with repeated malaria infections. PLoS Pathog. 2010;6:e1000912.
37. Jeurissen A, Ceuppens JL, Bossuyt X.T lymphocyte dependence of the antibody response to 'T lymphocyte independent type 2'antigens. Immunology. 2004;111:1-7

38. Walker PS, Reid ME. The Gerbich blood group system: a review. Immunohematology. 2010;26:60-5.

39. Salomao $M$, Zhang $X$, Yang $Y$, Lee $S$, Hartwig JH, Chasis JA, et al. Protein 4.1R-dependent multiprotein complex: new insights into the structural organization of the red blood cell membrane. Proc Natl Acad Sci USA. 2008;105:8026-31.

40. Cooling L. Blood groups in infection and host susceptibility. Clin Microbiol Rev. 2015;28:801-70

41. Wassmer SC, Carlton JM. Glycophorins, blood groups, and protection from severe malaria. Trends Parasitol. 2016;32:5-7.

42. Maier AG, Duraisingh MT, Reeder JC, Patel SS, Kazura JW, Zimmerman PA, et al. Plasmodium falciparum erythrocyte invasion through glycophorin $\mathrm{C}$ and selection for Gerbich negativity in human populations. Nat Med. 2003;9:87-92.

43. Serjeantson SW. A selective advantage for the Gerbich-negative phenotype in malarious areas of Papua New Guinea. P N G Med J. 1989;32:5-9.

44. Lin E, Tavul L, Michon P, Richards JS, Dabod E, Beeson JG, et al. Minimal association of common red blood cell polymorphisms with Plasmodium falciparum infection and uncomplicated malaria in Papua New Guinean school children. Am J Trop Med Hyg. 2010;83:828-33.

45. Patel SS, Mehlotra RK, Kastens W, Mgone CS, Kazura JW, Zimmerman PA The association of the glycophorin C exon 3 deletion with ovalocytosis and malaria susceptibility in the Wosera, Papua New Guinea. Blood. 2001:98:3489-91.

46. Fowkes FJ, Michon P, Pilling L, Ripley RM, Tavul L, Imrie HJ, et al. Host erythrocyte polymorphisms and exposure to Plasmodium falciparum in Papua New Guinea. Malar J. 2008;7:1.

47. Schofield L, Vivas L, Hackett F, Gerold P, Schwarz RT, Tachado S. Neutralizing monoclonal antibodies to glycosylphosphatidylinositol, the dominant TNF-alpha-inducing toxin of Plasmodium falciparum: prospects for the immunotherapy of severe malaria. Ann Trop Med Parasitol. 1993:87:617-26.

48. Schofield L, Novakovic S, Gerold P, Schwarz RT, McConville MJ, Tachado SD. Glycosylphosphatidylinositol toxin of Plasmodium up-regulates intercellular adhesion molecule-1, vascular cell adhesion molecule-1, and E-selectin expression in vascular endothelial cells and increases leukocyte and parasite cytoadherence via tyrosine kinase-dependent signal transduction. J Immunol. 1996;156:1886-96.

49. Mbenque B, Diatta B, Niang B, Diagne N, Ndiaye M, Marrama L, et al. Differential kinetics of plasma procalcitonin levels in cerebral malaria in urban Senegalese patients according to disease outcome. Microb Res. 2011;2:80-4.

50. Boutlis CS, Fagan PK, Gowda DC, Lagog M, Mgone CS, Bockarie MJ, et al. Immunoglobulin $\mathrm{G}$ (lgG) responses to Plasmodium falciparum glycosylphosphatidylinositols are short-lived and predominantly of the lgG3 subclass. J Infect Dis. 2003:187:862-5.

\section{Submit your next manuscript to BioMed Central and we will help you at every step:}

- We accept pre-submission inquiries

- Our selector tool helps you to find the most relevant journal

- We provide round the clock customer support

- Convenient online submission

- Thorough peer review

- Inclusion in PubMed and all major indexing services

- Maximum visibility for your research

Submit your manuscript at www.biomedcentral.com/submit

C BioMed Central 